Research article

\title{
New distributional records in lichen family Graphidaceae for Andhra Pradesh, India
}

\author{
Satish Mohabe ${ }^{1}$, Anjali Devi B. ${ }^{1}$, A. Madhusudhana Reddy ${ }^{1}$, \\ Gangadhar Pandhava ${ }^{1}$ and Sanjeeva Nayaka ${ }^{2}$ \\ ${ }^{1}$ Department of Botany, Yogi Vemana University, Vemanapuram, Kadapa-516003, Andhra Pradesh, India \\ ${ }^{2}$ Lichenology Laboratory, CSIR-National Botanical Research Institute, Rana Pratap Marg, Lucknow-226001, \\ Uttar Pradesh, India
}

*Corresponding Author: grassced@yahoo.com

[Accepted: 22 September 2017]

\begin{abstract}
The present paper reports 15 lichen taxa viz. Chapsa leprocarpoides, Diploschistes scruposus, Fissurina capsulata, F. cingalina, F. comparimuralis, F. egena, Glyphis cicatricosa, Graphis cincta, G. crebra, G. duplicata, G. furcata, G. handelii, G. leptocarpa, G. lineola and Thecaria austroindica of family Graphidaceae for the first time from Andhra Pradesh. Out of which, genera Chapsa, Fissurina and Thecaria are recorded for the first time from the state. The brief description and updated distribution are provided for all the taxa studied.
\end{abstract}

Keywords: Taxonomy - Graphidaceae - Thelotremoid - New addition - Rayalaseema forest.

[Cite as: Mohabe S, Anjali DB, Reddy AM, Pandhava G \& Nayaka S (2017) New distributional records in lichen family Graphidaceae for Andhra Pradesh, India. Tropical Plant Research 4(3): 383-390]

\section{INTRODUCTION}

The lichen family Graphidaceae is well known for its tropical and subtropical distribution in the world. The family was monographed by Staiger (2002) which has been recently reclassified on the basis of molecular phylogeny (Plata $e t$ al. 2012, Lücking et al. 2017). At present Graphidaceae is the largest family comprising of 2,161 species in the world and 406 species in India (Singh \& Sinha 2010). In the last five years, several taxa under the family have been described from the various regions of India (Chitale et al. 2011, Sharma et al. 2012, Joshi et al. 2012, Singh \& Singh 2014, Mohabe et al. 2015, 2016a). Some of the studies especially focused to know the diversity of the family within the different states of the country (Gupta \& Sinha 2012, Singh \& Singh 2015). In recent lichenological investigations on Rayalaseema region of Andhra Pradesh, Anantapur, Chittoor and YSR districts additions have been made in lichen diversity of the region (Anjali et al. 2013, Mohabe et al. 2016b, 2017). So far seven species viz. Diorygma junghuhnii (Mont. \& Bosch) Kalb \& al., D. kurnoolensis Mohabe, Nayaka \& Reddy, Diploschistes scruposus (Schreb.) Norman, D. actinostomus (Pers. ex Ach.) Zahlbr., Graphis neeladriensis Mohabe, Anjali \& Nayaka, G. plumierae Vain. and G. subalbostriata Lücking are known from the state under Graphidaceae. The present investigation on Graphidaceous lichens reports new distributional records as well as addition to the diversity of Graphidaceae within Rayalaseema forests of Andhra Pradesh.

\section{MATERIALS AND METHODS}

A total of 54 samples of Graphidaceous lichens were segregated from Herbarium Department of Botany, Yogi Vemana University, Kadapa, Andhra Pradesh. The lichens were mostly observed on tree barks, which were collected from Tirumala hills, Talakona mango orchards and Shikaram between altitudes ranges of 500 to $1000 \mathrm{~m}$ in Chittoor and Kurnool district respectively. These collections were made by the authors during 20122014. The morphological features of lichen thallus and lirellae (ascomata) were observed under Leica S8AP0 stereo-zoom microscope. Spot test for colour reaction were carried out by $10 \%$ aqueous solution of potassium hydroxide (K), Steiner's stable para-phenylenediamine solution (PD) and calcium hypochlorite solution (C). Light microscope of Leica DM500 was used for anatomical investigation of lirellae. All the measurements of anatomical structures were taken in water and $\mathrm{K}$ while Lugol's iodine solution was used especially for 
hymenium, ascus and ascospores observation. The lichen substances were identified with Thin Layer Chromatography in solvent system 'A' following White \& James (1985) and Orange et al. (2001). The other literatures were followed for identification includes Makhija \& Adawadkar (2007), Lücking et al. (2009) Sharma et al. (2012), Joshi et al. (2012), Lücking et al. (2017) was consulted for nomenclature and classification.

\section{RESULTS}

Taxonomic details and Indian distribution of endemic lichens of Graphidaceae in Andhra Pradesh, India
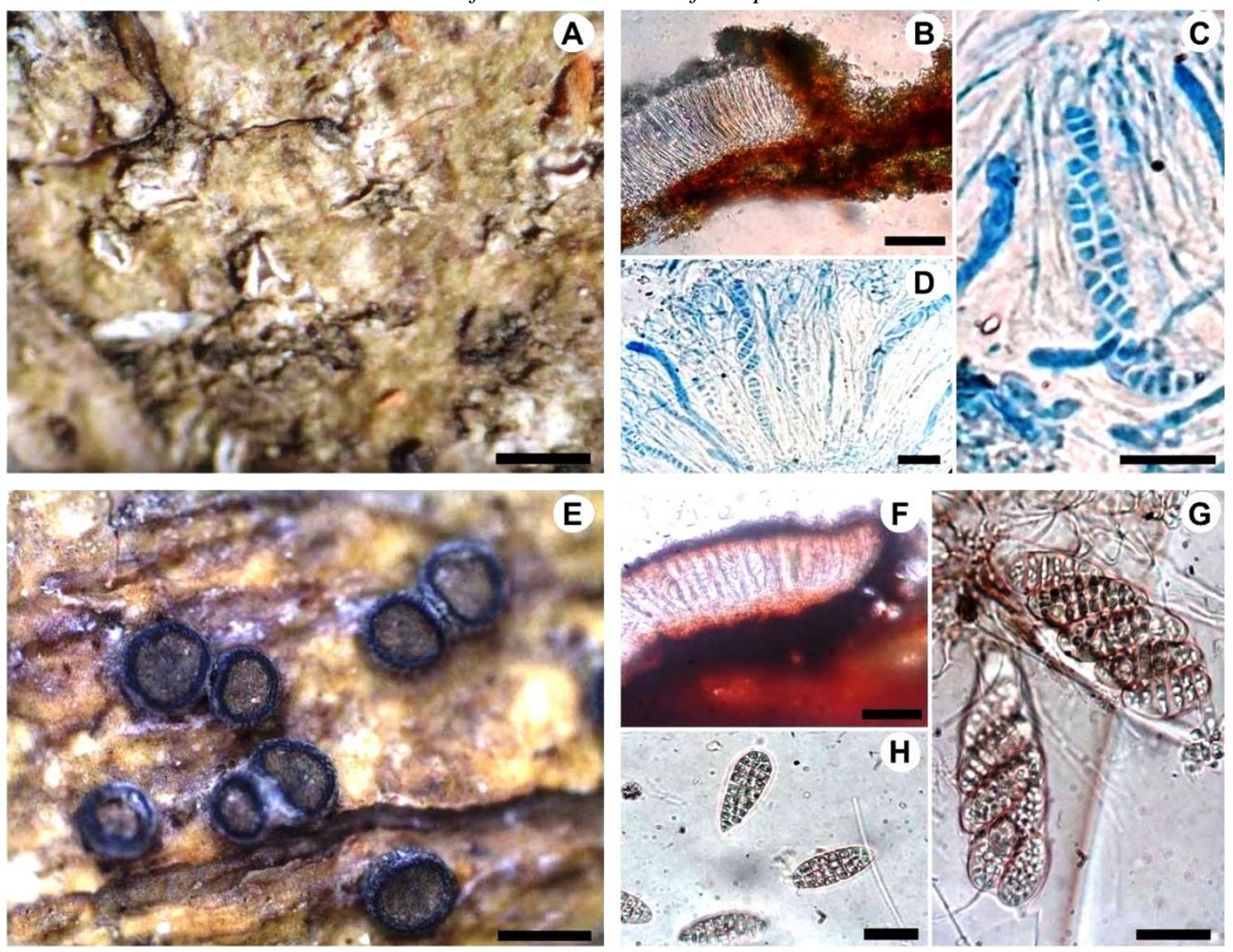

Figure 1. Endemic lichens of Graphidaceae in Andhra Pradesh, India: (A-D), Fissurina capsulata Makhija \& Adaw. [A, Habit (Scale: $0.5 \mathrm{~mm}$ ); B, V.s. of lirellae (Scale: $50 \mu \mathrm{m}$ ); C-D, Ascospores within ascus in lectophenol cotton blue (Scale: $25 \mu \mathrm{m})$ ]; (E-H), Thecaria austroindica (D. D. Awasthi \& Upreti) K. P. Singh \& G.P Sinha [E, Habit (Scale: $0.5 \mathrm{~mm}$ ); F, V.s. of apothecia showing completely carbonized exciple (Scale: $100 \mu \mathrm{m}$ ); G, Ascus with 8-spored ascospores (Scale: 25 $\mu \mathrm{m}), \mathbf{H}$, Brown muriform ascospores (Scale: $25 \mu \mathrm{m}$ )].

1. Fissurina capsulata Makhija \& Adaw., Lichenologist 39(2): 171. 2007. (Figs. 1A-D)

This corticolous species is characterized by yellowish brown, thin, ecorticated thallus; simple to branched, $0.5-1.0 \mathrm{~mm}$ long ascomata, margin with swollen tissue; yellowish brown exciple; clear hyaline hymenium; 8-spored ascus; hyaline, transversely 3-5-septate, 11-16 $\times 4-5 \mu \mathrm{m}$, halonate ascospores and lacking lichen substances.

Distribution in India: The species is endemic to India and it was earlier reported from Tamil Nadu (Singh \& Sinha 2010).

Specimen examined: Andhra Pradesh, Chittoor district, Talakona, on bark of Mangifera indica, $539 \mathrm{~m}$, 16.03.2013, ADB \& SM 3638 (YVUH); Kurnool district, Shikaram, 7 km before Srisailam, on bark, 745 m, 12.01.2013, SM \& KSR 3001 (YVUH).

2. Thecaria austroindica (D. D. Awasthi \& Upreti) K. P. Singh \& G.P Sinha, Indian Lichens: An Annotated Checklist, 242, 2010. (Figs. 1E-H)

This corticolous species is characterized by epiphloedal, buff yellow to brownish, evanescent, smooth to cracked thallus; rounded to elongate or oval, scattered, emergent, $0.2-0.5 \mathrm{~mm}$ apothecia; margin thin, concolorous with thallus; entire, slightly irregular towards outside, divergent labia covered with thalline 
layer; brown black, pruinose, dusty, open, concave disc; completely carbonized exciple; hyaline, inspersed hymenium; 2-8 spored ascus; brown, muriform, transversely 4-8 septate, longitudinally 1-3 septate, I+ red brown ascospores; lacking lichen substances.

Distribution in India: The species is endemic to India and it was earlier reported from Arunachal Pradesh, Assam, Karnataka, Manipur and Odhisa (Singh \& Sinha 2010, Devi et al. 2015).

Specimens examined: Andhra Pradesh, Chittoor district, Tirumala hills, on bark, 917 m, 06.02.2013, ADB \& SM 3251 (YVUH); Tirumala hills, Dharmagiri, on bark, 937m, 07.02.2013, SM \& ADB 3435 (YVUH); Shilathoranam, on bark of roadside tree, 973 m, 07.02.2013, ADB \& SM 3331 3251, 3332, 3298, 3318, 3340 (YVUH); on bark, 958 m, 05.07.2014, SM \& ADB 4059, 4051 (YVUH); Neeladri range, viewpoint, on bark, 06.07.2014, SM \& ADB 4160, 4168 (YVUH); Starting of Tirumala hills, upside, on bark, 917 m, 06.02.2013, $A D B \& S M 3242($ YVUH).

Taxonomic details and Indian distribution of new lichen records of Graphidaceae for Eastern Ghats

3. Fissurina egena (Nyl.) Nyl. Staiger, Biblioth. Lichenol. 85: 136. 2002. (Figs. 2A-C)

This corticolous species is characterized by greenish brown to olive brown, smooth to cracked, sometimes verruculose thallus; $0.5-1.5 \mathrm{~mm}$ long, immersed, simple to furcate, straight to flexuous, irregularly branched, ascomata surrounded by white roof like structure; epruinose, slit like disc; entire, noncarbonized, convergent exciple; hyaline, clear, I- hymenium; indistinct, hyaline hypothecium; immature ascus and ascospores; simple paraphyses and lacking lichens substances.

Distribution in India: It was earlier known from West-Bengal (Jagadeesh et al. 2006).

Specimen examined: Andhra Pradesh, Kurnool district, Shikaram, 7 km before Srisailam, on bark, 745 m, 12.01.2013, SM \& KSR 3014 (YVUH).

\section{Graphis crebra Vain. Hedwigia 38: 256. 1899. (Figs. 2D-G)}

This corticolous species is characterized by smooth to cracked, whitish grey, $3-5 \mathrm{~cm}$, corticated thallus; white medulla, with large crystals at the base of exciple; indistinct to brownish prothallus; lirellate ascomata erumpent, short to elongate, $0.5-2.0 \mathrm{~mm}$ long, $0.1-0.5 \mathrm{~mm}$ wide, branched, with lateral thalline margin; entire, black, epruinose labia, exposed disc with white pruina; laterally carbonized 14-25 $\mu \mathrm{m}$ thick exciple; pale brown, 12-17 $\mu \mathrm{m}$ thick epihymenium; hyaline, inspersed, 85-173 wide, 68-95 $\mu \mathrm{m}$ high, I- hymenium; hyaline, 17-35 $\mu \mathrm{m}$ high hypothecium; hyaline, transversely 4-9-septate, 16-33 $\times 4-10 \mu \mathrm{m}$ ascospores and contains norstictic acid.

Distribution in India: It was earlier reported from West-Bengal (Jagadeesh et al. 2012).

Specimens examined: Andhra Pradesh, Chittoor district, Talakona, on bark of Mangifera indica, $539 \mathrm{~m}$, 16.03.2013, $A D B$ \& SM 3645, 3749 (YVUH); Tirumala hills, Neeladri range, on bark, 06.07.2014, SM \& $A D B 4074$ (YVUH).

\section{Graphis furcata Fée, Essai Crypt. Ecorc.: 40. 1824. (Figs. 2H-K)}

This corticolous species is characterized by whitish grey, rough to cracked, ecorticated thallus with black prothallus; short to elongate, erumpent, $0.1-0.2 \mathrm{~mm}$ long, $0.2-0.3 \mathrm{~mm}$ wide lirellate ascomata, simple to partially branched with acute ends, flexuous lirellae laterally covered by the thallus; concealed disc; entire, often thinly white-pruinose labia; laterally carbonized exciple; clear, hyaline hymenium; 8-spored ascus; hyaline, ellipsoid to fusiform, transversely 5-11 septate, 22-38 $\times 8-9 \mu \mathrm{m}$ ascospores and thallus lacking lichen substances.

Distribution in India: It was earlier reported from Andaman \& Nicobar Islands and Meghalaya (Singh \& Sinha 2010, Singh \& Singh 2015).

Specimens examined: Andhra Pradesh, Chittoor district, Tirumala hills, Dharmagiri, on bark, $937 \mathrm{~m}$, 07.02.2013, SM \& ADB 3427, 3442 (YVUH); Shilathoranam, on bark, $937 \mathrm{~m}, 07.02 .2013, S M \& A D B 3315$ (YVUH).

\section{Graphis leptocarpa Fée, Essai Crypt. Exot. 36: 1825. (Figs. 2L-O)}

This crustose corticolous species is characterized by whitish grey, rough to cracked, $2 \mathrm{~cm}$ across thallus with indistinct prothallus; lirellate ascomata erumpent, short and sparsely branched $0.5-1.0 \mathrm{~mm}$ long, 0.01$0.05 \mathrm{~mm}$ wide, with acute ends, with lateral thalline margin, slit like disc; entire, epruinose labia; laterally carbonized, 19-48 $\mu \mathrm{m}$ thick exciple; greyish brown, 18-28 $\mu \mathrm{m}$ epihymenium; hyaline, inspersed, 73-106 $\mu \mathrm{m}$ high, I- hymenium; hyaline, 29-56 $\mu \mathrm{m}$ hypothecium; hyaline, 53-66 × 12-19 $\mu \mathrm{m}, 8$-spored ascus; 

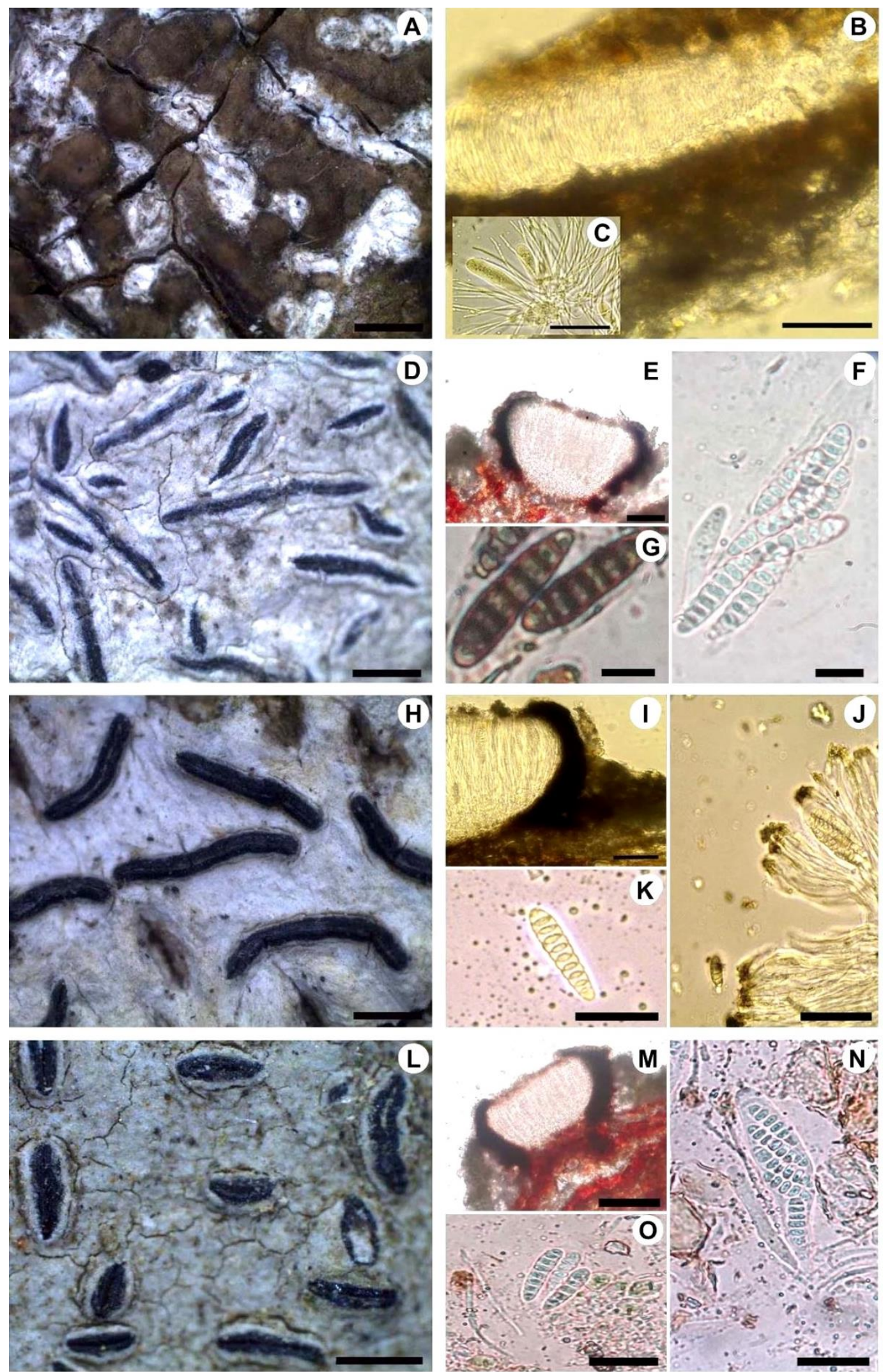

Figure 2. New records for Eastern Ghats: (A-C), Fissurina egena (Nyl.) Nyl. [A, Habit (Scale: $0.5 \mathrm{~mm}$ ); B, Clear hymenium (Scale: $50 \mu \mathrm{m}$ ); C, Ascus (Scale: $50 \mu \mathrm{m}$ )]; (D-G), Graphis crebra Vain. [D, Habit (Scale: $0.5 \mathrm{~mm}$ ); E, Inspersed hymenium with lateral carbonization (Scale: $50 \mu \mathrm{m}$ ); F, Ascospores in K (Scale: $10 \mu \mathrm{m}$ ); G, Ascopores in I (Scale: $10 \mu \mathrm{m})$ ]; (H-K), Graphis furcata Fée [H, Habit (Scale: $0.5 \mathrm{~mm}$ ); I, Clear hymenium with entire labia and lateral thalline margin (Scale: $50 \mu \mathrm{m}$ ); J, Ascus with ascospores and pyraphyses (Scale: $50 \mu \mathrm{m}$.); K, Ascospore (Scale: $25 \mu \mathrm{m}$ )]; (L-O), Graphis leptocarpa Fée [L, Habit (Scale: $0.5 \mathrm{~mm}$ ); M, Entire labia with lateral thalline margin (Scale: $50 \mu \mathrm{m}$ ); N, Ascospores within ascus in $\mathrm{K}$ (Scale: $25 \mu \mathrm{m})$; $\mathbf{O}$, ascopores in $\mathrm{K}$ (Scale: $25 \mu \mathrm{m})$ ]. 
colourless, transversely 7-11-septate, 27-32 × 6-7 $\mu \mathrm{m}$ ascospores; paraphyses 1.6-3.8 $\mu \mathrm{m}$ thick, contains stictic and constictic acid.

Distribution in India: It was earlier reported from Assam, Jammu \& Kashmir (Daimari et al. 2014, Goni \& Sharma 2015).

Specimen examined: Andhra Pradesh, Chittoor district, Talakona, before jungle thrills, on bark of Mangifera indica, $539 \mathrm{~m}, 16.03 .2013, A D B \& S M 3743$ (YVUH).

Taxonomic details and Indian distribution new lichen records of Graphidaceae for Andhra Pradesh
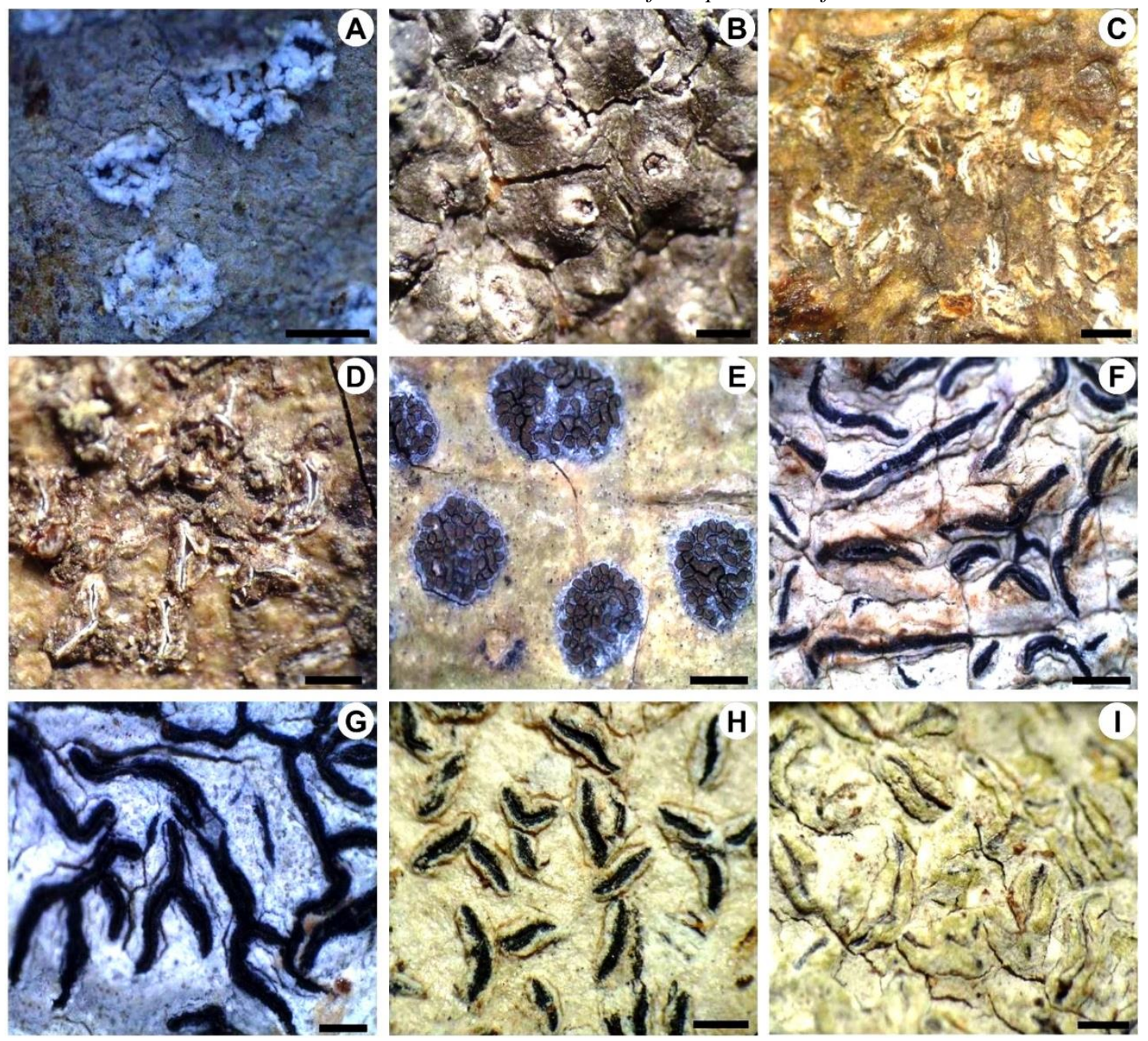

Figure 3. New records for Andhra Pradesh: A, Chapsa leprocarpoides (Hale) Cáceres \& Lücking (Scales: $0.5 \mathrm{~mm}$ ); B, Diploschistes scruposus (Schreb.) Norman (Scales: $0.5 \mathrm{~mm}$ ); C, Fissurina cingalina (Nyl.) Staiger (Scale: $0.1 \mathrm{~mm}$ ); D, Fissurina comparimuralis Staiger (Scale: $0.1 \mathrm{~mm}$ ); E, Glyphis cicatricosa Ach. (Scales: $0.5 \mathrm{~mm})$; F, Graphis cincta (Pers.) Aptroot (Scales: $0.5 \mathrm{~mm}$ ); G, Graphis duplicata Ach. (Scales: $0.2 \mathrm{~mm}$ ); H, Graphis handelii Zahlbr. (Scales: $0.5 \mathrm{~mm}$ ); I, Graphis lineola Ach. (Scales: $0.5 \mathrm{~mm}$ ).

7. Chapsa leprocarpoides (Hale) Cáceres \& Lücking, Libri Bot. 22: 52, 2007. (Fig. 3A)

This species is characterized by corticolous habitat, pale olive to fawn coloured, ecorticate thallus; immersed to semi-immersed, rounded to angular, flesh coloured, 0.05-0.2 mm apothecia; pruinose disc and low uneven to lobed margins; a free, pale to brownish or hyaline proper exciple; $2-4$ spored, $40-60 \times 20-30$ $\mu \mathrm{m}$ ascus; hyaline muriform, oval-ellipsoidal, 20-25 $\times 10-15 \mu \mathrm{m}$ ascospores and lacking lichen substances.

Distribution in India: It was earlier reported from Karnataka (Joshi et al. 2012).

Specimen examined: Andhra Pradesh, Chittoor district, Japali Anjaneya Swamy Temple, on bark, 746.5 m, 13.06.2012, SN \& AMR 1819 (YVUH).

\section{Diploschistes scruposus (Schreb.) Norman, Nyt Mag. Naturvidensk. 7: 232, 1853. (Fig. 3B)}

This is a saxicolous species characterized by greenish grey to brown, rimose-areolate to cracked, upto 3 $\mathrm{cm}$ across, epruinose thallus; thin to thick, $0.5-1.5 \mathrm{~mm}$ areolae, plane to subconvex at ostiolar region; 
urceolate, subimmersed to sessile, $0.5-1.0 \mathrm{~mm}$, slightly pruinose ascomata; dark brown, concave disc; hyaline hymenium; cylindricoclavate asci; brown, muriform, ellipsoid, 26-38 $\times 11-19 \mu \mathrm{m}$, transversely 4-7, vertically 1-3 septate ascospores with longitudinal septa and contains diploschistesic, lecanoric and orsellinic acid.

Distribution in India: It was earlier reported from Himachal Pradesh, Jammu \& Kashmir, Madhya Pradesh, Maharashtra, Meghalaya, Sikkim, Tamil Nadu, Uttarakhand, West Bengal (Singh \& Sinha 2010).

Specimen examined: Andhra Pradesh, Chittoor district, Tirumala hills, starting point, top of the hill, on rock, 917 m, 06.02.2013, ADB \& SM 3176 (YVUH).

\section{Fissurina cingalina (Nyl.) Staiger, Biblioth. Lichenol. 85: 128, 2002. (Fig. 3C)}

This corticolous species is characterized by greenish grey to greenish brown, smooth to cracked, glossy thallus delimited by black hypothallus; $1.0-2.5 \mathrm{~mm}$ long, branched, immersed, terminally acute lirelline ascomata; epruinose, slit like disc; entire, non-carbonized, convergent exciple; hyaline, clear, I- hymenium; indistinct, hyaline hypothecium; 8 -spored ascus; hyaline, muriform, transversely $6-9$, vertically $2-3$ septate, 25-30 × 9-16 $\mu \mathrm{m}$, halonate ascospores; simple paraphyses and lacking lichens substances.

Distribution in India: It was earlier reported from Kerala, Maharashtra, Manipur and Tamil Nadu (Singh $\&$ Sinha 2010, Singh \& Singh 2015).

Specimens examined: Andhra Pradesh, Chittoor district, Talakona, on bark of Mangifera indica, $539 \mathrm{~m}$, 16.03.2013, $A D B \& S M$ 3699, 3709 (YVUH); Shilathoranam, on roadside tree bark, $958 \mathrm{~m}, 05.07 .2014$, $A D B \& S M 4030(\mathrm{YVUH})$.

\section{Fissurina comparimuralis Staiger. Biblioth. Lichenol. 85: 134, 2002. (Fig. 3D)}

This corticolous species is characterized by yellowish to dark brown, slightly verruculose to cracked, slightly glossy thallus; delimited by thin black hypothallus; $0.3-1.0 \mathrm{~mm}$ long, simple to irregularly branched, curved, immersed, terminally acute lirelline, ascomata with slightly raised thalline margin; immersed, slit-like disc; entire, non-carbonized, orange, convergent exciple; hyaline, clear, I- hymenium; indistinct hypothecium; simple paraphyses; 8-spored asci; hyaline, submuriform, transversely 5-6 and longitudinally 1-2-septate, 21-24 ×5-8 $\mu \mathrm{m}$, halonate, I+ weak blue ascospores and lacking lichen substances.

Distribution in India: It was earlier known from Kerala (Sharma et al. 2012).

Specimen examined: Andhra Pradesh, Chittoor district, Talakona, on bark of Mangifera indica, $539 \mathrm{~m}$, 16.03.2013, ADB \& SM 3638 (YVUH); Kurnool district, Shikaram, 7 km before Srisailam, on bark, $745 \mathrm{~m}$, 12.01.2013, SM \& KSR 3013 (YVUH).

\section{Glyphis cicatricosa Ach. Syn. Meth. Lich.: 107. 1814. (Fig. 3E)}

This corticolous species is characterized by greenish to yellowish grey or yellowish brown, smooth thallus; irregular lirellate apothecia embedded in carbonaceous stroma; dark brown, concave disc; hyaline, clear, 65-125 $\mu \mathrm{m}$ high hymenium, simple paraphyses; 8-spored ascus; transversely 5-10 septate, halonate, I+ blue, 25-65 x 8-12 $\mu \mathrm{m}$ ascospores with rounded lumina, lacking lichen substances.

Distribution in India: It was earlier reported from Andaman \& Nicobar Islands, Goa, Karnataka, Kerala, Sikkim, Tamil Nadu, West Bengal, Manipur, Meghalaya and Nagaland (Singh \& Sinha 2010, Singh \& Singh 2015).

Specimens examined: Andhra Pradesh, Chittoor district, Tirumala hills, Dharmagiri, on bark, $937 \mathrm{~m}$, 07.02.2013, $S M \& A D B 3440$ (YVUH); on road side tree bark, $958 \mathrm{~m}, 05.07 .2014, A D B \& S M 4035,4045$, 4029, 4033 (YVUH); Shilathoranam, on bark, 937 m, 07.02.2013, SM \& ADB 3318, 3332 (YVUH); Srivari padalu, on bark, $1076 \mathrm{~m}, 07.02 .2013, A D B \& S M 3526,3535$ (YVUH).

\section{Graphis cincta (Pers.) Aptroot in A.W. Archer, Fl. Australia 57: 651. 2009. (Fig. 3F)}

This corticolous species is characterized by thin, smooth, whitish grey thallus; lirellate, $0.1-0.3 \mathrm{~mm}$ wide, $0.2-1.2 \mathrm{~mm}$ long ascomata; epruinose, slit like disc; epruinose, entire labia, sometimes broad at apex; laterally carbonised, 19-44 $\mu \mathrm{m}$ thick exciple; distinct, brown to dark brown, 15-20 $\mu \mathrm{m}$ thick epihymenium; inspersed with oil globules, 56-79 $\mu \mathrm{m}$ high hymenium; hyaline, 19-22 $\mu \mathrm{m}$ high hypothecium; hyaline, clavate, 59-79 $\times 5-10 \mu \mathrm{m}, 8$-spored ascus; hyaline, transversely 4-8-septate, 20-25 $\times 4-8 \mu \mathrm{m}$, I+ blue ascospores and contains norstictic acid. 
Distribution in India: It was earlier reported from Arunachal Pradesh, Goa, Karnataka, Kerala, Maharashtra, Manipur, Meghalaya, Nagaland and Tamil Nadu (Singh \& Sinha 2010, Singh \& Singh 2015).

Specimens examined: Andhra Pradesh, Chittoor district, Tirumala hills, Dharmagiri, on the way, on bark, 937 m, 07.02.2013, ADB \& SM 3422 (YVUH); Shilathoranam, on bark of road side tree, $958 \mathrm{~m}, 05.07 .2014$, $S M \& A D B 4029$ (YVUH).

\section{Graphis duplicata Ach. Syn. Meth. Lich.: 81. 1814. (Fig. 3G)}

This corticolous species is characterized by greyish white, smooth to rough, corticated thallus with brown to black prothallus; lirellate ascomata, erumpent to prominent, $0.2-1.0 \mathrm{~mm}$ long, $0.05-0.1 \mathrm{~mm}$ wide, with indistinct to basal thalline margin, elongate and irregularly branched, striate labia; laterally carbonized exciple; clear hymenium; hyaline, transversely 7-11-septate, 13-42 $\times 5-8 \mu \mathrm{m}$ ascospores and lacking lichen substances.

Distribution in India: It was earlier reported from Arunachal Pradesh, Karnataka, Kerala, Maharashtra, Manipur, Meghalaya, Nagaland, Tamil Nadu and Uttarakhand (Singh \& Sinha 2010, Singh \& Singh 2015).

Specimens examined: Andhra Pradesh, Chittoor district, Thamballapalli, near Shiva temple, on bark, 900 m, 05.01.2013, SM \& AMR 2833, 2834 (YVUH).

14. Graphis handelii Zahlbr. in Hand.-Mazz., Symb. Sin. 3: 39 \& 40. 1930. (Fig. 3H)

This corticolous species is characterized by greyish white, smooth to cracked or areolate, corticated thallus; lirellate apothecia erumpent, short to elongate and sparsely to irregularly branched, with lateral thalline margin; epruinose, exposed disc; entire labia; laterally carbonized exciple; hyaline, inspersed hymenium; clavate, 8-spored ascus, transversely 5-11-septate, 16-46 $\times 5-10 \mu \mathrm{m}$ ascospores and contains norstictic acid.

Distribution in India: It was earlier reported from Manipur, Meghalaya and West Bengal (Singh \& Sinha 2010, Singh \& Singh 2015).

Specimen examined: Andhra Pradesh, Chittoor district, Tirumala hills, Kousthubham Cottage, $859 \mathrm{~m}$, 06.02.2013, $A D B$ \& SM 3159 (YVUH).

\section{Graphis lineola Ach. Lichenogr. Universalis: 264. 1810. (Fig. 3I)}

This corticolous species is characterized by greenish grey to yellowish green, rough to cracked, lirellate apothecia erumpent, with lateral to complete thalline margin; concealed disc; entire, epruinose labia; laterally carbonized exciple; inspersed hymenium; 8-spored ascus; hyaline, transversely 5-11-septate, 22-42 $\times 6-9 \mu \mathrm{m}$ ascospores and lacking lichen substances.

Distribution in India: It was earlier reported from Arunachal Pradesh, Jammu \& Kashmir, Karnataka, Manipur, Tamil Nadu, Uttarakhand, Uttar Pradesh and West Bengal-hills (Singh \& Sinha 2010, Goni \& Sharma 2015, Devi et al. 2015, Gupta \& Sinha 2016).

Specimen examined: Andhra Pradesh, Chittoor district, Tirumula hills, Kousthubham cottage, on bark, $859 \mathrm{~m}, 06.02 .2013, A D B \& S M 3172,3162$ (YVUH).

\section{CONCLUSION}

At present Graphidaceae is the third largest family in Andhra Pradesh with 22 species under 7 genera which is followed by families Physciaceae and Parmeliaceae (Mohabe 2016a). Among these Fissurina capsulata Makhija \& Adaw. and Thecaria austroindica (D. D. Awasthi \& Upreti) K. P. Singh \& G.P Sinha recorded as endemic to India. The study also recorded four species viz. Fissurina egena (Nyl.) Nyl., Graphis crebra Vain., G. furcata Fée and G. leptocarpa Fée are found first time from southern part (Eastern Ghats) of India. Further, the present study on lichen diversity of Andhra Pradesh would represent 167 species, while Chittoor district alone has 92 species. The available information on Graphidaceous lichens will helpful as base line records to lichen flora of Andhra Pradesh and can be used for biomonitoring studies in future.

\section{ACKNOWLEDGEMENTS}

The first author is very grateful to University Grant Commission, New Delhi (F./PDFSS-2015-17-MAD12168) and Department of Science \& Technology, New Delhi and CSIR, New Delhi (Letter No. 60(0100)/11/ EMR - II, 2012 for financial assistance; the Director and Dr. D.K. Upreti, Chief Scientist, CSIR-National Botanical Research Institute, Lucknow for providing infrastructure facilities of Lichenology Laboratory for lichens identification; Mr. K. Suresh Raju for helping in lichen collection and to the Forest official of Andhra 
Pradesh to permit for sample collections.

\section{REFERENCES}

Anjali DB, Mohabe S, Reddy MA, Nayaka S \& Chandramati PS (2013) Diversity and distribution of lichens in YSR District, Andhra Pradesh with several new additions. Indian Journal of Plant Sciences 2(4): 1-9.

Chitale G, Makhija U \& Sharma B (2011) Additional species of Graphis from Maharashtra, India. Mycotaxon 115: 469-480.

Daimari R, Hazarika N, Hoque RR, Nayaka S \& Upreti DK (2014) New Records of Epiphytic Lichens from Three districts of Assam, India. Indian Forester 140(8): 807-811.

Devi RKS, Rout J, Upreti DK, Nayaka S \& Pinokiyo A (2015) New records of lichens from Manipur State, North-eastern India. Mycosphere 6(6): 796-813.

Goni R \& Sharma N (2015) Additions to lichen flora of Jammu and Kashmir, India. Tropical Plant Research 2(2): 78-81.

Gupta P \& Sinha GP (2012) A new record of lichen in the genus Graphis for India from Assam. Indian Journal of Forestry 35(1): 133-134.

Gupta P \& Sinha GP (2016) New Records of Lichen Taxa from Uttar Pradesh, India. Cryptogam Biodiversity and Assessment 1(2): 64-69.

Jagadeesh Ram TAM, Sinha GP, Singh KP \& Borthakur SK (2006) Four species of lichens new to Indian lichen flora. Indian Journal of Forestry 30(4): 535-538.

Jagadeesh Ram TAM, Sinha GP \& Singh KP (2012) Lichen Flora of Sundarbans Biosphere Reserve, West Bengal. Bishen Singh Mahendra Pal Singh, Dehradun.

Joshi S, Upreti DK \& Nayaka S (2012) The lichen genus Chapsa (Graphidaceae) in India. Myxotaxon 120: 23-33.

Lücking R, Archer AW \& Aptroot A (2009) A worldwide key to the genus Graphis (Ostropales: Graphidaceae). Lichenologist 41: 363-452.

Lücking R, Brendan P Hodkinson \& Steven DL (2017) The 2016 classification of lichenized fungi in the Ascomycota and Basidiomycota - Approaching one thousand genera. The Bryologist 119(4): 361-416.

Makhija U \& Adawadkar B (2007) Trans-septate species of Acanthothecis and Fissurina from India. The Lichenologist 39(2): 165-185.

Mohabe S, Anjali DB, Nayaka S \& Reddy AM (2016a) New species and new records of Graphis (Ostropales: Graphidaceae) from Eastern Ghats, India. Tropical Plant Research 3(3): 611-615.

Mohabe S, Anjali DB, Reddy AM \& Nayaka S (2017) Floristic assessment of lichens diversity in Anantapur district of Andhra Pradesh. Cryptogam Biodiversity and Assessment 2(1): 01-07.

Mohabe S, Anjali DB, Reddy AM, Nayaka S \& Chandramati PS (2016b) An appraisal of lichen biota in Chittoor district of Andhra Pradesh, India. In: Pullaiah T \& Sandhya Rani (eds) Biodiversity in India. Regency Publications, New Delhi.

Mohabe S, Nayaka S, Reddy MA \& Anjali DB (2015) Diorygma kurnoolensis (Graphidaceae), a new species of saxicolous lichen from Southern India. Geophytology 45(1): 47-50.

Orange A, James PW \& White FJ (2001) Microchemical methods for the identification of lichens. British Lichen Society, U.K.

Plata ER, Lücking R \& Lumbsch TH (2012) A new classification for the family Graphidaceae (Ascomycota: Lecanoromycetes: Ostropales). Fungal Diversity 52: 107-121.

Sharma BO, Khadikar P \& Makhija U (2012) New Species and new contributions in the lichen genera Fissurina and Hemithecium from India. The Lichenologist 44(3): 339-362.

Singh KP \& Sinha GP (2010) Indian Lichens: Annotated Checklist. Botanical Survey of India, Kolkata.

Singh P \& Singh KP (2014) Two new species of Graphis (Ascomycota: Ostropales: Graphidaceae), from IndoBurma biodiversity hotspot. Mycosphere 5(4): 504-509.

Singh P \& Singh KP (2015) Additional lichen records of Graphidaceae for Manipur, Meghalaya and Nagaland, North-East India. Geophytology 45(2): 181-194.

Staiger B (2002) Die Flechtenfamilie Graphidaceae: Studien in Richtung einer natürlicheren Gliederung. Bibliotheca Lichenologica 85: 1-526.

White FJ \& James PW (1985) A new guide to microchemical techniques for the identification of lichen substances. Bulletin British Lichen Society 57: 1-41. 